\title{
东南极拉斯曼丘陵含假蓝宝石紫苏辉石 石英岩的发现及其地质意义*
}

全来喜 刘小汉 徐平 韩秀伶 (中国科学院地质研究所岩石圈构造演化开放实验室, 北京 100029)
赵越 任留东王彦斌

(中国地质科学院地质研究所, 北京 100037)

\section{关链词东南极 拉斯曼丘陵 含假蓝宝石紫苏辉石石英岩}

本文报道了东南极拉斯曼丘陵地区首次发现的含假蓝宝石紫苏辉石石英岩, 对其中假蓝 宝石矿物进行了岩相学、矿物学及变质作用研究, 并进一步探讨了其形成的地质意义.

\section{1 地质背景}

东南极拉斯曼丘陵主要由米洛、布洛克尼斯和斯托尼斯三大半离及众多岛礁组成(图 1), 面积约 $60 \mathrm{~km}^{2}$, 属于东南极广泛分布的晚元古高级变质地体的一部分. 角闪岩相至麻粒岩相 的变泥质岩、变砂岩、石英岩、混合岩化副片麻岩、长英质正片麻岩及镁铁质麻粒岩构成该区主 要露头.

一般认为, 该区在 $1000 \mathrm{Ma}$ 左右经 历低压麻粒岩相变质作用, 其峰期温压条 件为 $0.45 \mathrm{GPa}$ 和 $750^{\circ} \mathrm{C}$, 以后发生近等 温减压 $0.05 \sim 0.1 \mathrm{GPa}^{[1]}$. 近年来认为, 该区主要由早期残留的 $\mathrm{D}_{1}$ 麻粒岩相变质 构造 (1000 Ma) 和一系列后期的 $D_{2}-D_{6}$ 角闪岩相-麻粒岩相变质构造 $(500 \mathrm{Ma})$ 组 成 ${ }^{[2]}$. 其中后期的 $500 \mathrm{Ma}$ (泛非) 事件以 低中压变质作用为特征, 具有顺时针的 $P-T$ 演化轨迹 ${ }^{[3,4]}$. Carson 等 $^{[3]}$ 报道了 $\mathrm{M}_{2}$ 具有 $0.7 \mathrm{GPa}$ 和 $780^{\circ} \mathrm{C}$ 的峰期变质条 件(Grt-Opx 温压计), 只有 Ren 等 ${ }^{[4]}$ 报道

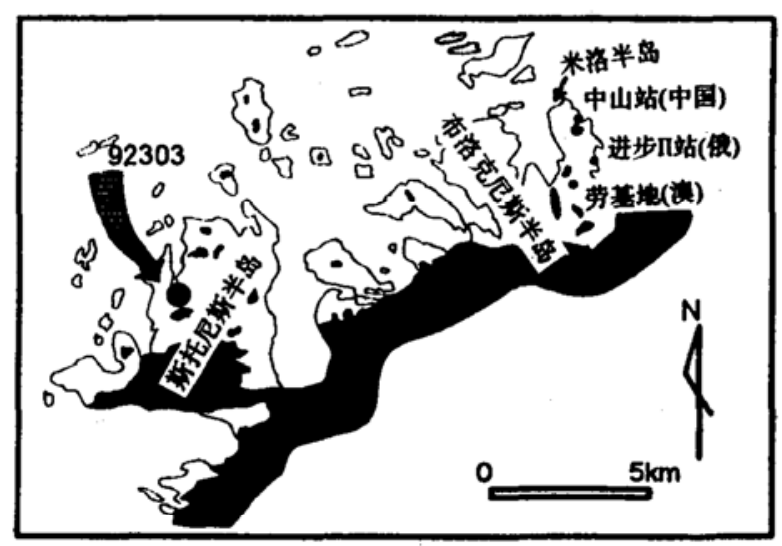

图 1 东南极拉斯曼丘陵地区含假蓝宝石紫苏辉石 石英岩产出位置图 了早期 $\mathrm{M}_{1}$ 具有 $0.9 \mathrm{GPa}$ 和 $850^{\circ} \mathrm{C}$ 的峰期变质温压条件 (Grt-Pl-Sil- Qtz 压力计和 Opx-Cpx 温度 计). 最近, Tong 和 $\mathrm{Liu}^{1}{ }^{1}$ 报道了早期 $\mathrm{M}_{1}$ 更高的峰期变质条件 $0.95 \mathrm{GPa}$ 和 $870{ }^{\circ} \mathrm{C}$ (Grt-Opx 温压计).

1995-08-29 收稿, 1995-12-25 收修改稿

* 国家自然科学基金和国家“八五”科技攻关资助项目

1)Tong L, Liu X. The prograde metamorphism of the Larsemann Hills, East Antarctica: evidence for an anticlockwise P-T path. Terra Antartica, Italy, 1996 (in press) 


\section{2 岩相学特征}

含假蓝宝石紫苏辉石石英岩样品 92303 采于斯托尼斯半岛西北部(图 1). 岩石呈暗灰色, 以 $20 \mathrm{~cm}$ 左右的夹层产于层状的黑云母柱晶石紫苏辉石堇青石片麻岩中. 该岩石主要由粗粒 的黑云母 $(20 \% \pm)$ 、石英 $(45 \% \pm)$ 以及较粗大的紫苏辉石变斑晶 $(30 \% \pm)$ 组成, 变斑晶大小一 般为 $2 \sim 3 \mathrm{~cm}$. 另外, 还有少量的磁铁矿、尖晶石、矽线石等矿物, 锆石、磷灰石、独居石为副矿 物, 具不等粒变晶结构, 紫苏辉石变晶具笁状变晶结构.

假蓝宝石则与石英、尖晶石和磁铁矿一起作为紫苏辉石变斑晶的包裹体赋存, 同时在假蓝 宝石中又有残留的尖晶石包裹体存在, 在磁铁矿中也有矽线石包裹体(图 2). 此外, 紫苏辉石 变晶中还有尖晶石 + 矽线石 + 磁铁矿的包裹体, 以及黑云母和石英包裏体, 其中黑云母和石英 包裹体大多数呈浑圆形. 紫苏辉石变晶中一些黑云母+石英包裹体的变形构造显然代表了该 区早期的 $\mathrm{D}_{1}$ 残留构造.

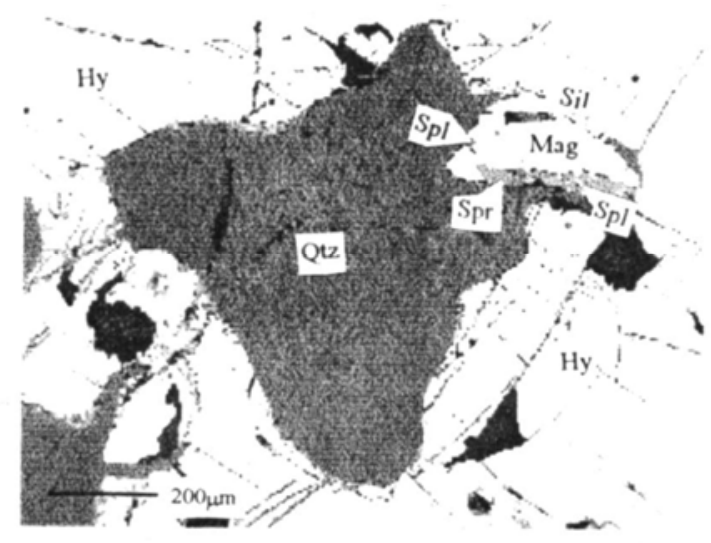

图 2 假蓝宝石 $(\mathrm{Spr})+$ 石英 $(\mathrm{Qtz})+$ 尖晶石 $(\mathrm{Spl})$ + 磁铁矿 $(\mathrm{Mag})$ 作为紫苏辉石 $(\mathrm{Hy})$ 变晶的 包裹体存在

在显微镜下, 该假蓝宝石呈自形的板条 状, 正中突起, 多色性明显, $N_{g}=$ 淡蓝, $N_{p}=$ 无色, 一级灰干涉色, 斜消光, $N_{g} \wedge c=57^{\circ}$. 紫苏辉石变晶也具有明显多色性, $N_{g}$ 淡 红, $N_{p}$ = 淡绿, 呈一级灰干涉色. 假蓝宝石 与石英及尖晶石和磁铁矿直接接触, 并共同 包于紫苏辉石变晶之中, 可推断假蓝宝石 + 石英 + 尖晶石 + 磁铁矿组合为残留的早期 $\mathrm{D}_{1}$ 矿物组合. 从其包裹关系推测, 假蓝宝石 在 $\mathrm{D}_{1}$ 期间通过如下变质反应形成: 尖晶石 + 石英 + 磁铁矿 $=$ 假蓝宝石. 该假蓝宝石 中含有尖晶石包裹体则指示了一个逆时针 近等压冷却的 $P-T$ 演化过程 ${ }^{[5]}$.

\section{3 矿物学及变质作用的 P-T 条件}

本文中所有矿物化学成分的探针分析, 是在中国科学院地质研究所岩石圈构造演化开放 实验室的 CAMECA SX-51 电子探针仪上完成. 加速电压 $15 \mathrm{kV}$, 样品电流 (在 $\mathrm{Al}_{2} \mathrm{O}_{3}$ 上) $20 \mathrm{nA}$.

该岩石主要矿物化学成分的探针分析结果列于表 1 . 假蓝宝石的理想化学分子式为 $\mathrm{Mg}_{4} \mathrm{Al}_{8} \mathrm{Si}_{2} \mathrm{O}_{20}$, 其中经常有 $\mathrm{Fe}^{2+}$ 代替 $\mathrm{Mg}$ 和 $\mathrm{Fe}^{3+}$ 替代 $\mathrm{Al}$ 及 $\mathrm{Al}$ 替换 $\mathrm{Si}^{[6]}$. 对该假蓝宝石进行 了 15 个点的成分分析, 其中选取两个代表性的结果与 Napier 杂岩中的假蓝宝石成分进行对 比并显示成分上的一致性 (表 1). 该假蓝宝石成分从总体上来看, 其成分显示不均匀的特点. 其 $X_{\mathrm{Mg}}\left(\mathrm{Mg} / \mathrm{Mg}+\mathrm{Fe}^{2+}\right.$ ) 值在 $0.705 \sim 0.724$ 之间, 略低于 Napier 杂岩中假蓝宝石的 $X_{\mathrm{Mg}}$ 值 $(0.746)^{[7]}$.

尖晶石的 $\mathrm{Al}_{2} \mathrm{O}_{3}(62.45 \%)$ 和 $\mathrm{MgO}(13.01 \%)$ 含量, 高于本区已报道的泥质片麻岩中尖晶 石矿物的 $\mathrm{Al}_{2} \mathrm{O}_{3}(53.41 \%)$ 和 $\mathrm{MgO}(2.89 \%)^{[4]}$, 它的 $X_{\mathrm{Mg}}$ 值为 0.526 . 紫苏辉石富铝, 其 $\mathrm{Al}_{2} \mathrm{O}_{3}$ 
含量为 $6.70 \% \sim 7.07 \%$, 其 $X_{\mathrm{Mg}}$ 值为 $0.68 \sim 0.69$. 矽线石 $\mathrm{FeO}$ 含量相对较高, 可达 $1.64 \%$. 黑云母为金云母成分, 其 $\mathrm{MgO}$ 含量达 $19.02 \%$,相应的 $X_{\mathrm{Mg}}$ 值为 0.762 .

表 1 拉斯曼丘陵含假蓝宝石紫苏辉石石英岩主要矿物的探针分析结果 ${ }^{a)}$

\begin{tabular}{crrrrrrr}
\hline & $\mathrm{Spl}$ & \multicolumn{1}{c}{$\mathrm{Spr}$} & \multicolumn{1}{c}{$\mathrm{Spr}$} & \multicolumn{1}{c}{$\mathrm{Spr}$} & $\mathrm{Hy}$ & $\mathrm{S}$ il & $\mathrm{Phl}$ \\
\hline $\mathrm{SiO}_{2}$ & 0.079 & 11.575 & 11.721 & 12.76 & 49.428 & 36.434 & 38.758 \\
$\mathrm{TiO}_{2}$ & 0.012 & 0.009 & 0.79 & 0.36 & 0.078 & 0.040 & 2.269 \\
$\mathrm{Al}_{2} \mathrm{O}_{3}$ & 62.448 & 61.307 & 60.839 & 60.49 & 7.070 & 61.575 & 14.545 \\
$\mathrm{Cr}_{2} \mathrm{O}_{3}$ & 0.206 & 0.037 & 0.122 & 0.00 & 0.076 & 0.030 & 0.019 \\
$\mathrm{Fe}_{2} \mathrm{O}_{3}$ & 2.214 & - & - & - & 1.720 & - & - \\
$\mathrm{MgO}$ & 12.012 & 14.828 & 14.698 & 15.96 & 22.904 & 0.219 & 19.021 \\
$\mathrm{CaO}$ & 0.000 & 0.007 & 0.002 & 0.00 & 0.039 & 0.000 & 0.000 \\
$\mathrm{MnO}$ & 0.228 & 0.041 & 0.037 & 0.00 & 0.088 & 0.007 & 0.000 \\
$\mathrm{FeO}$ & 20.921 & 10.462 & 10.965 & 9.69 & 18.195 & 1.635 & 10.575 \\
$\mathrm{NiO}$ & 0.187 & 0.000 & 0.042 & - & 0.020 & 0.000 & 0.128 \\
$\mathrm{Na}_{2} \mathrm{O}$ & 0.027 & 0.024 & 0.008 & 0.00 & 0.000 & 0.001 & 0.375 \\
$\mathrm{~K}_{2} \mathrm{O}$ & 0.000 & 0.000 & 0.007 & 0.00 & 0.000 & 0.000 & 9.407 \\
平均 & 99.836 & 98.290 & 98.520 & 99.26 & 99.618 & 99.941 & 95.097 \\
$X_{\mathrm{Mg}_{8}}$ & 0.526 & 0.716 & 0.705 & 0.746 & 0.692 & & 0.762 \\
\hline
\end{tabular}

a) $\mathrm{Spr}{ }^{*}$ 为 Napier 杂岩中假蓝宝石 (据 Hensen 和 Motoyoshi ${ }^{[7]}$ ), Spl 为尖晶石, $\mathrm{Spr}$ 为假蓝宝石, $\mathrm{Hy}$ 变紫苏辉石, Sil 为矽 线石, $\mathrm{Phl}$ 为金云母, “ - ”为末测

由以上可以看出, 该岩石各矿物的 $X_{\mathrm{Mg}}$ 值按假蓝宝石 $(0.72)$ 一紫苏辉石 $(0.69)$ 一尖晶石 (0.53) 顺序降低, 但金云母的 $X_{\mathrm{M}_{\mathrm{g}}}$ 值 $(0.76)$ 为最 高. 这暗示了该岩石原岩可能为富镁的含泥质 砂岩, 且其矿物组合受原岩成分和形成时的温压 条件制约.

由于该岩石紫苏辉石变晶缺乏堇青石和石 榴子石矿物组合包裹体, 所以无法用矿物温压计 来定量计算早期 $M_{1}$ 期间假蓝宝石形成的 $P-T$ 条件. 然而, 与该岩石互层的黑云母柱晶石紫苏 辉石堇青石片麻岩中紫苏辉石变晶里的镁堇青 石包裹体获得了 $0.75 \mathrm{GPa}$ 和 $850^{\circ} \mathrm{C}$ 的 $\mathrm{M}_{1}$ 峰期 $P-T$ 条件(作者未发表资料), 这一结果应该制约 了假蓝宝石形成 $P-T$ 条件的下限, 而该区 Grt$O p x$ 温压计获得的最高的 $M_{1} P-T$ 条件值 0.95 $\mathrm{GPa}$ 和 $850^{\circ} \mathrm{C}$ 可能制约了其形成条件的上限 ${ }^{1)}$. 这样, 假蓝宝石形成的 $P-T$ 范围应为: $P=0.75$

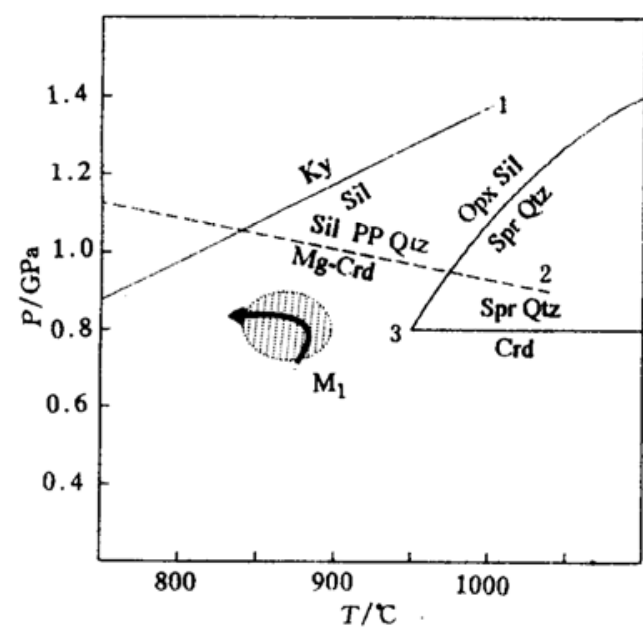

图 3 假蓝宝石组合的变质反应及 $P-T$ 稳定域图 1 一据 Holdaway ${ }^{[8]}, 2$ 一据 Holdaway 和 Lee ${ }^{[9]}$, 3 -一据 $\mathrm{Grew}^{[10]}$ $-0.95 \mathrm{GPa}, T=850-870^{\circ} \mathrm{C}$. 但是, 由堇青石高温高压实验变质反应可以看出, 假蓝宝石开 始出现的温度为 $950^{\circ} \mathrm{C}$ (图 3). 因此, 该假蓝宝石形成的温度上限可高至 $950^{\circ} \mathrm{C}$.

1) 见 1205 页脚注 


\section{4 假蓝宝石组合形成的地质意义}

本文首次报道了东南极拉斯曼丘陵含假蓝宝石组合紫苏辉石石英岩. 研究结果表明, 假 蓝宝石、石英、尖晶石和磁铁矿作为紫苏辉石变晶的包裹体存在,代表了该区早期残留的 $M_{1}$ 矿物组合. 假蓝宝石通过如下变质反应形成: 尖晶石 + 石英 + 磁铁矿 $=$ 假蓝宝石. 变质的温 压条件为: $P=0.75 \sim 0.95 \mathrm{GPa}, T=850 \sim 950^{\circ} \mathrm{C}$. 从变质反应和温压条件来看, 假蓝宝石的 形成发生在高温和干的变质条件下. 假蓝宝石中含有尖晶石包裹体指示了一个逆时针近等压 冷却的 $P-T$ 轨迹 $[5]$. 这一逆时针近等压冷却的 $P-T$ 轨迹, 反映了拉斯曼丘陵地区的早期 $\mathrm{M}_{1}$ $(1000 \mathrm{Ma})$ 变质热事件与岩浆垫板作用有关, 并伴随陆壳的伸展. 这一结论也与该区最近的 研究结果一 $\mathrm{M}_{1}$ 进变质作用一致 ${ }^{1)}$

致谢对叶大年院士、从柏林教授、王恺怡、间月华、王清晨和陈海泓研究员、B.J.Hensen 教授和 S. L. Harley 及 Y. Motoyoshi 博士所给与的帮助表示感谢.

\section{参考文献}

1 Stüwe K, Powell R. Low pressure granulite facies metamorphism in the Larsemann Hills area, East Antarctica: petrology and tectonic implications for the evolution of the Prydz Bay area. J Metamorphic Geol, 1989, 7:465 483

2 Dirks P H G M, Carson C J, Wilson C J L. The deformational history of the Larsemann Hills, Prydz Bay: the importance of the the Pan-African (500 Ma) in East Antarctica. Antarctic Sci, 1993, 5:179 192

3 Carson C J, Dirks P H G M, Hand M et al. Compressional and extensional tectontics in low-medium pressure granulites from the Larsemann Hills, East Antarctica. Geol Mag, 1995, 132:151 170

4 Ren L, Zhao Y, Liu X et al . Re-examination of the metamorphic evolution of the Larsemann Hills, East Antarctica. Recent Progress on Antarctic Earth Science, TERRAPUB, Tokyo, 1992, 145 153

5 Harley S L, Hensen B J. Archaean and Proterozoic high-grade terranes of East Antarctica (40-80.E): a case study of diversity in granulite facies metamorphism. In: Ashworth J R, Brown M eds. High-T Metamorphism and Crustal Anataxis. London: Unwin Hyman, 1990. 320 370

6 Deer W A, Howie R A, Zussman J. Rock-forming Minerals. Vol. 2A. Single-chain Silcates. London: Longman, 1978

7 Hensen B J, Motoyoshi Y. Osumilite-producing reactions in high temperature granulites from the Napier Complex, East Antarctica: tectonic implications. Recent Progress on Antarctic Earth Science, TERRAPUB, Tokyo, 1992. 87 92

8 Holdaway M J. Stability of andalusite and the aluminium silicate phase diagram. Am J Sci, 1971, 271:97 131

9 Holdaway M J, Lee S M. Fe-Mg cordierite stability in high-grade pelitic rocks based on experimental, theoretical, and natural observations. Contrib Mineral Petrol, 1977, 63:175 198

10 Grew E S. Sapphirine + quartz association from Archaean rocks in Enderby Land, Antarctica. Am Mineral, 1980, 65:821 836

1) 见 1205 页脚注. 